\title{
Fabrication, Characterization, and Simulation of Glass Devices with AIN Thin-Film Transducers for Excitation of Ultrasound Resonances
}

\author{
André G. Steckel,,$^{1, *}$ Henrik Bruus $\odot,{ }^{1, \dagger}$ Paul Muralt, $, 2,+\frac{\dagger}{}$ and Ramin Matloub ${ }^{3, \S}$ \\ ${ }^{1}$ Department of Physics, Technical University of Denmark, DTU Physics Building 309, Kongens Lyngby DK-2800, \\ Denmark \\ ${ }^{2}$ Materials Science, EPFL, Station 12, Lausanne 1015, Switzerland \\ ${ }^{3}$ PIEMACS Sàrl, EPFL Innovation Parc, Bâtiment C, Lausanne 1015, Switzerland
}

(Received 16 November 2020; revised 2 April 2021; accepted 27 May 2021; published 7 July 2021)

\begin{abstract}
We present the fabrication of $570-\mu \mathrm{m}$-thick millimeter-sized soda-lime-silicate float-glass blocks with a 1- $\mu \mathrm{m}$-thick AIN thin-film piezoelectric transducer sandwiched between thin metallic electrodes and deposited on the top surface. The electromechanical properties are characterized by electrical-impedance measurements in the frequency range from 0.1 to $10 \mathrm{MHz}$ with a peak-to-peak voltage of $0.5 \mathrm{~V}$ applied to the electrodes. We measure the electrical-impedance spectra of 35 devices, all of width $2 \mathrm{~mm}$, but with nine different lengths ranging from 2 to $6 \mathrm{~mm}$ and with two to seven copies of each individual geometry. Each impedance spectrum exhibits many resonance peaks, and we carefully measure the five most prominent ones in each spectrum. We compare the resulting 173 experimental resonance frequencies with simulation results from a finite-element-method model that we develop. When we use the material parameters from the manufacturer, we obtain an average relative deviation of the 173 simulated resonance frequencies from the experimental values of $(-4.6 \pm 0.1) \%$. When we optimize the values of the Young's modulus and Poisson's ratio of the float glass in the simulation, this relative deviation decreases to $(-0.5 \pm 0.1) \%$. Our results suggest a method for an accurate in situ determination of the acoustic parameters at ultrasound frequencies of any elastic solid onto which a thin-film transducer can be attached.
\end{abstract}

DOI: 10.1103/PhysRevApplied.16.014014

\section{INTRODUCTION}

Aluminum nitride (AlN) is one of the most commonly used materials in integrated thin-film piezoelectric transducers for actuating microelectromechanical systems (MEMS). Because of their low dielectric and mechanical loss tangents, their structural and chemical stability, and their compatibility with standard silicon-based CMOS microfabrication techniques, AlN sputtered thin films are commercially used in thin-film bulk-wave acousticresonator filters [1]. The academic literature reports applications of AlN thin-film transducers as rf filters [2], contour-mode resonators [3], switches [4,5], suspended microchannel resonators [6], and accelerometers [7].

\footnotetext{
*angust@fysik.dtu.dk

†bruus@fysik.dtu.dk

†paul.muralt@epfl.ch

\$ramin.matloub@piemacs.ch
}

Published by the American Physical Society under the terms of the Creative Commons Attribution 4.0 International license. Further distribution of this work must maintain attribution to the author(s) and the published article's title, journal citation, and DOI.
Detailed studies of AlN-thin-film-actuated high-tone bulk acoustic resonators with high quality factors have been performed in the $0.3-3 \mathrm{GHz}$ range on 350- $\mu \mathrm{m}$-thick substrates of sapphire, crystal quartz, fused silica, and silicon [8], and on 30- $\mu$ m-thick Si membranes $[9,10]$.

Because AlN is a nonferroelectric polar material, reorientation of the polar axis is not possible. The material growth process therefore has to provide a textured structure that includes the alignment of the polar directions [11], since otherwise the random orientations of the grains constituting the AlN thin film would result in a zero average piezoelectric effect. In the work presented here, we therefore follow the process developed in our previous work on pure [12] and Sc-doped [13,14] AlN films, and select $\mathrm{Pt} / \mathrm{Ti}$ as the seed layer for the bottom electrode stack as it promotes nucleation of (002)-oriented AlN grains.

Over the past few years, piezoelectric thin films made of nonferroelectric polar materials, such as zinc oxide $(\mathrm{ZnO})$ and AlN, have found a broad range of lab-on-a-chip applications such as biosensing, particle or cell concentrating, sorting, patterning, pumping, mixing, nebulization, and jetting. Integrated devices with acoustic transducers, sensors, and microfluidic channels have been fabricated by depositing such piezoelectric films onto a wide range of materials 
beyond the usual silicon substrates, such as ceramics, diamond, glass, and, more recently, also polymers, metallic foils, and flexible glass, for making flexible devices. Such thin-film acoustic-wave devices have great potential for implementing integrated, disposable, or bendable lab-on-a-chip devices into various sensing and actuating applications [15]. To advance such applications, we focus in the present work on AlN thin-film transducers deposited on glass substrates and driven in the $0.1-10 \mathrm{MHz}$ range.

The deposition of AlN thin films and the control of their texture are more difficult than in the case of $\mathrm{ZnO}$ films [16], and their integration into MEMS is not a trivial task, as they tend to have a high in-plane stress with a sharp transition around zero stress [17]. However, the thermal conductivity of AIN is more than 3 times larger than of $\mathrm{ZnO}$, which enables the operation of AlN films at a higher power level, an important feature for applications in rf technology. AlN films are normally sputter deposited at moderate temperatures (typically $200-400^{\circ} \mathrm{C}$ ) to achieve optimal performance, although "room temperature" deposition with a local temperature near the target of around $150^{\circ} \mathrm{C}$ is sometimes used [18]. Also, the deposition conditions, especially the amount of oxygen or moisture in the vacuum chamber, have significant effects on the growth and microstructure of AlN films. Growing AlN films thicker than a few micrometers is particularly challenging because of the potentially large film stress and the tendency to form microcracks.

In the present work, we use our titanium-seed-layer technique [12] to improve the fabrication of AIN thin-film transducers deposited on glass substrates for future use in biosensing and lab-on-a-chip applications. In Sec. II, we describe the design of the devices, followed in Sec. III by a presentation of the fabrication and characterization processes. In Sec. IV, we then present the characterization of the acoustic properties of the devices by measuring electrical-impedance spectra in the frequency range from 0.1 to $10 \mathrm{MHz}$. A numerical model and its finite-elementmethod implementation is described in Sec. V. A comparison of the simulation results with the results of the experiments presented in Sec. VI shows such good agreement that our numerical handling of the measured impedance spectra can be turned into an improved in situ determination of the acoustic properties of the glass substrate. Finally, concluding remarks are presented in Sec. VII.

\section{DEVICE DESIGN}

Figure 1 shows the design of the devices under study. This design is chosen to ease the microfabrication, the experimental characterization, and the subsequent validation of a finite-element method model of the system. All devices consist of a simple box-shaped $570-\mu$ m-thick and 2-mm-wide block of soda-lime-silicate (SLS) float glass [19] of length $L$ diced from a wafer. $L$ is in the range
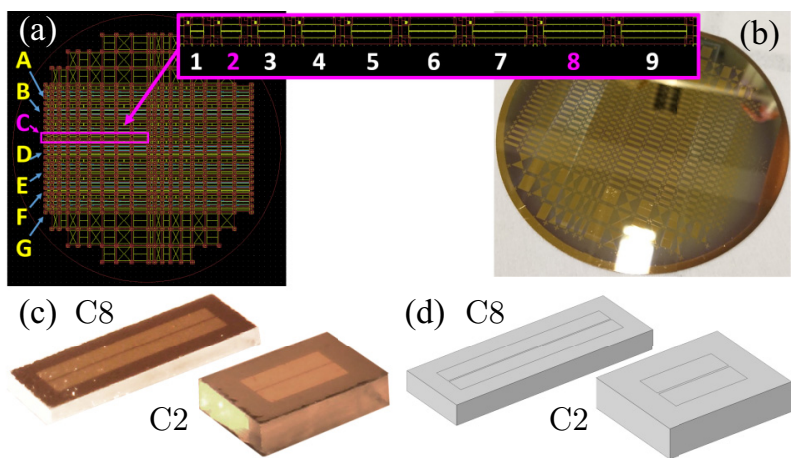

FIG. 1. (a) Design of the electrode mask for the lithography step in the fabrication process that defines the gold electrodes on the top surface of the glass wafer. The relevant devices are taken from rows $\mathrm{A}-\mathrm{G}$ and columns $1-9$. The inset shows row $\mathrm{C}$, columns $1-9$. Devices with the same column index are nominally identical. (b) Photograph of the fabricated wafer before dicing it into individual devices. (c) Microscope images of the actual devices $\mathrm{C} 2$ and $\mathrm{C} 8$. (d) Rendering of the two 2-mm-wide and 0.57-mm-high devices C2 (2.5 mm long) and C8 (5.5 mm long), showing the outline of the 1-mm-wide top electrode split into two halves by a $40-\mu \mathrm{m}$-wide gap.

from 2 to $6 \mathrm{~mm}$ in steps of $0.5 \mathrm{~mm}$, thus resulting in nine groups of nominally identical devices. A $1-\mu \mathrm{m}$-thick AlN thin-film piezoelectric transducer, sandwiched between submicrometer-thick metal electrodes, is deposited on the top surface of the glass block. To ease the dicing process and to avoid short-circuiting between the two electrodes, the top electrode of the transducer has its width reduced to $W_{\mathrm{TE}}=1 \mathrm{~mm}$ and its length to $L_{\mathrm{TE}}=L-1 \mathrm{~mm}$. Moreover, to allow antisymmetric voltage actuation, the top electrode is split in half by a $40-\mu \mathrm{m}$-wide gap perpendicular to the length direction. As listed in Table I, there are up to seven nominally identical copies, A, B, C, D, E, F, and $\mathrm{G}$, in each group of devices.

TABLE I. A list of the 35 devices under study. The rectangular glass blocks are all $0.57 \mathrm{~mm}$ thick and have a nominal width $W=2 \mathrm{~mm}$ and a length $L$ in the range from 2 to $6 \mathrm{~mm}$. The deposited AIN thin-film piezoelectric transducer is $1 \mu \mathrm{m}$ thick and is sandwiched between a $0.1-\mu \mathrm{m}$-thick bottom and a 0.15 $\mu \mathrm{m}$-thick top metal electrode. The top electrode has a total width $W_{\mathrm{TE}}=1 \mathrm{~mm}$ and length $L_{\mathrm{TE}}=L-1 \mathrm{~mm}$. All devices with the same column index are nominally identical.

\begin{tabular}{lccc}
\hline \hline Device column & Device row & $L(\mathrm{~mm})$ & $L_{\mathrm{TE}}(\mathrm{mm})$ \\
\hline 1 & C, D, G & 2.0 & 1.0 \\
2 & C, D, E, F, G & 2.5 & 1.5 \\
3 & A, B, C, G & 3.0 & 2.0 \\
4 & A, B, D & 3.5 & 2.5 \\
5 & A, B, C & 4.0 & 3.0 \\
6 & B, C & 4.5 & 3.5 \\
7 & A, B, C, D, E & 5.0 & 4.0 \\
8 & A, B, C, D, E, F, G & 5.5 & 4.5 \\
9 & C, D, E & 6.0 & 5.0 \\
\hline \hline
\end{tabular}




\section{FABRICATION AND CHARACTERIZATION OF THE PIEZOELECTRIC THIN-FILM TRANSDUCER}

The device fabrication is based on a 4-in. SLS float-glass wafer (Schott Suisse SA, Yverdon). This glass has a low thermal expansion, a high thermal-shock resistance, and an ability to withstand temperatures up to $490{ }^{\circ} \mathrm{C}$ for long periods, so by not raising the process temperature above $300^{\circ} \mathrm{C}$, we ensure high quality and reproducibility in our fabrication process. Following a microfabrication process similar to the one described in Ref. [12], and sketched in Fig. 2, the first step is cleaning of the glass substrate with isopropyl alcohol and acetone to remove organic contamination. Additional plasma cleaning is performed immediately before the deposition of the bottom electrode in the deposition tool. We prepare textured Ti, Pt, and AlN thin films using a dedicated cluster sputtering chamber (Pfeiffer Vacuum), and then sputter-deposit $10 \mathrm{~nm}$ of Ti at $300{ }^{\circ} \mathrm{C}$ to serve as an adhesion layer between the glass substrate and the Pt bottom electrode. Next, the 100-nm-thick Pt electrode is deposited with the same tool in a different chamber, also at $300^{\circ} \mathrm{C}$, without breaking the vacuum. Subsequently, $1 \mu \mathrm{m}$ of (0001)-textured AlN is deposited using a dedicated reactive pulsed direct-current magnetron sputtering chamber at $300^{\circ} \mathrm{C}$, and on top of the AlN film $10 \mathrm{~nm}$ of $\mathrm{Cr}$ and $150 \mathrm{~nm}$ of $\mathrm{Au}$ are sputter deposited as the top electrode, with $\mathrm{Cr}$ being used as the adhesion layer between the AlN and Au. In the next process step, standard photolithography is exploited to transfer the intended pattern onto the top electrode, and this pattern is realized by ion-beam etching (Veeco). Finally, the wafer is diced into smaller chips ready for use, as shown in Fig. 1(c). (a)

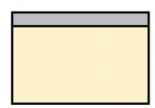

(d)

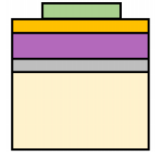

(b)

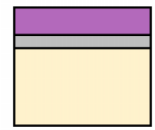

(e)

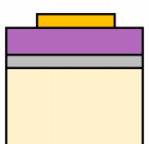

(c)

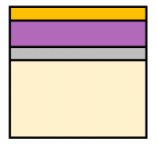

Photoresist Gold $(0.15 \mu \mathrm{m})$ AlN $(1 \mu \mathrm{m})$ Platinum $(0.1 \mu \mathrm{m})$ Glass $(570 \mu \mathrm{m})$
FIG. 2. Sketch of the fabrication process, where a stack consisting of a bottom electrode, an AlN thin film, and the top electrode is deposited on a glass block. (a) Deposition of a 10-nm-thick Ti adhesion layer (not shown) followed by a 100nm-thick platinum layer. (b) Deposition of the 1- $\mu$ m-thick AlN film. (c) Deposition of a 10-nm-thick $\mathrm{Cr}$ adhesion layer (not shown) followed by a 150-nm-thick gold layer. (d) Deposition and development of photoresist. (e) Patterning of the top electrode by ion-beam etching of the exposed gold, followed by removal of the photoresist.
After fabrication and dicing, the quality of the resulting devices is checked by visual inspection with a microscope, and their dimensions are measured using an electronic micrometer (RS Pro 705-1229, RS Components). By measuring all available devices, the average height and width are found to be $(571 \pm 1) \mu \mathrm{m}$ and $(2031 \pm 2) \mu \mathrm{m}$, respectively, and the average lengths of devices 1-9 are determined to be $(2011 \pm 1),(2512 \pm 1),(3013 \pm 1),(3514 \pm$ $1),(4016 \pm 1),(4500 \pm 1),(5016 \pm 1),(5509 \pm 1)$, and $(6018 \pm 1) \mu \mathrm{m}$, respectively. The uncertainties represent one standard deviation of several repeated measurements, and the relative uncertainty ranges from $0.2 \%$ for the height down to below $0.1 \%$ for the longest length.

\section{MEASURED ELECTRICAL IMPEDANCE}

The electrical impedance of the 35 devices listed in Table I is measured as a function of frequency from 0.1 to $3 \mathrm{MHz}$ in steps of $500 \mathrm{~Hz}$ using an Agilent 4294A Precision Impedance Analyzer (Agilent Technologies AG, Basel, Switzerland) with a $0.5-\mathrm{V}_{\mathrm{pp}}$ ac voltage applied in

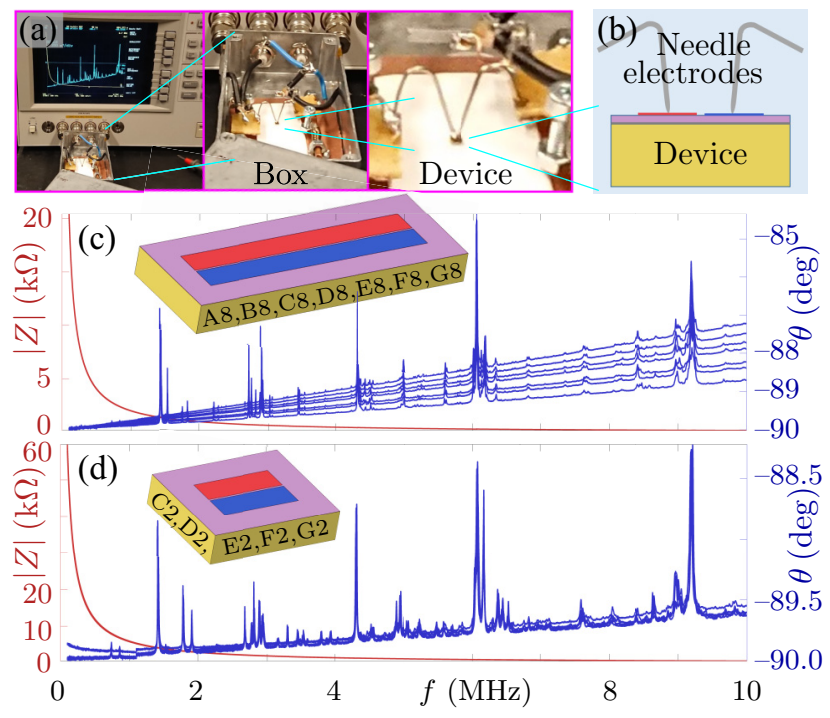

FIG. 3. (a) Three photographs of the spectrum analyzer and the attached metal box that provides electrical shielding of the device, but here with its lid removed to reveal the device mounted inside. An enlargement of the interior of the metal box shows the coaxial connectors and the wires leading to the device. A further enlargement shows the spring-loaded electrodes attached to the device. (b) End-view sketch of the spring-loaded electrodes attached to the device, consisting of a glass block (beige) and an AlN thin-film transducer (purple) with a split top electrode (red and blue). (c) Impedance spectra, showing the magnitude $|Z(f)|$ (red curves) and the phase $\theta(f)$ (blue curves) for the seven nominally identical devices A8, B8, C8, D8, E8, F8, and G8 shown in the inset. The phase curves differ, as the devices are not discharged initially. (d) As part (c) but for the five nominally identical devices C2, D2, E2, F2, and G2. Here, the phase curves are nearly identical, as the devices are discharged initially. 
antiphase between the two halves of the split top electrode. The bottom electrode is left floating.

To reduce the electrical noise, each device under study is placed in a grounded metal box, from where it is connected with short coaxial cables to the impedance analyzer by pressing spring-loaded needle electrodes onto each half of the split top electrode; see Figs. 3(a) and 3(b). The setup is also designed to minimize undesired parasitic components: the parasitic inductance in the system is reduced to somewhere around $L_{\text {ext }}=(28 \pm 15) \mathrm{nH}$, the parasitic resistance is roughly $R_{\mathrm{ext}}=(5 \pm 3) \Omega$, and the parasitic capacitance is negligible compared with the intrinsic capacitance $C_{\mathrm{dev}}$ of the device formed by the electrodes separated by the thin AlN film, ranging from $18 \mathrm{pF}$ for the smallest device to $86 \mathrm{pF}$ for the largest. This design ensures that the resonance frequency $f_{\mathrm{ext}}=(1 / 2 \pi)\left(L_{\mathrm{ext}} C_{\mathrm{dev}}\right)^{-(1 / 2)}$ involving the external inductance is pushed up in value, such that $100 \mathrm{MHz} \lesssim f_{\text {ext }} \lesssim 220 \mathrm{MHz}$, much larger than $10 \mathrm{MHz}$, and therefore does not interfere with the intrinsic resonances of the device under study. Finally, the device under study is placed on a folded soft piece of tissue paper to acoustically isolate it from the environment.

Examples of the 35 recorded impedance spectra $Z(f)=$ $|Z| e^{i \theta}$ are shown in Figs. 3(c) and 3(d), where we plot the magnitude $|Z(f)|$ and the phase $\theta(f)$. We first note that the intrinsic resonances of the devices are most clearly visible in the phase plots $\theta(f)$. Because of the parasitic impedances of the system, the base level of $\theta(f)$ shows a linear increase from the $-90^{\circ}$ of an ideal capacitor in the $\mathrm{dc}$ limit to a value between $-89.7^{\circ}$ and $-87.8^{\circ}$ at 10 $\mathrm{MHz}$. The device resonances appear as sharp peaks in the phase curve, with a width of around 0.01 times the resonance frequency itself and with an amplitude ranging from $0.1^{\circ}$ to $3.5^{\circ}$. This technique is a variant of standard resonant ultrasound spectroscopy (RUS) [20-22]. However, in contrast to conventional RUS, the ultrasound transducers in our case are an integrated but tiny part of the structures to be characterized.

Next, we note that the positions and amplitudes of the resonance peaks are nearly identical for samples with

TABLE II. For each of the nine device geometries, labeled by the column index $k=1-9$ in Table I, five resonance peaks $(k, n)$ of increasing frequency with $n=1-5$ are selected from the impedance spectrum for comparing the simulations with the measurements. Peaks $(4,1)$ and $(4,2)$, marked with asterisks, are not measured on device $\mathrm{B}$ in column $n=4$. The experimental value $f_{k, n}^{\text {exp }}$ is the average resonance frequency of peak $n$ over all devices (row indices A-G in Table I) with column index $k$. The simulation result $f_{k, n}^{\text {sim, } \mathrm{A}}$ is the corresponding resonance frequency obtained from the numerical modeling described in Sec. V using the parameter values given in the literature. The simulation result $f_{k, n}^{\text {sim,B }}$ is obtained by using the optimized values listed in Table IV of the Young's modulus and Poisson's ratio for the glass block. Finally, for $X=\mathrm{A}$ or $\mathrm{B}, \Delta_{k, n}^{X}=\left(f_{k, n}^{\operatorname{sim}, X}-f_{k, n}^{\exp }\right) / f_{k, n}^{\exp }$ is the relative deviation of the simulation result from the experimental value for resonance peak $n$ and device geometry $k$.

\begin{tabular}{|c|c|c|c|c|c|c|c|c|c|c|c|}
\hline $\begin{array}{l}\text { Peak } \\
k, n\end{array}$ & $\begin{array}{c}f_{k, n}^{\exp } \\
(\mathrm{MHz})\end{array}$ & $\begin{array}{l}f_{k, n}^{\operatorname{sim}, \mathrm{A}} \\
(\mathrm{MHz})\end{array}$ & $\begin{array}{l}f_{k, n}^{\operatorname{sim}, \mathrm{B}} \\
(\mathrm{MHz})\end{array}$ & $\begin{array}{l}\Delta_{k, n}^{\mathrm{A}} \\
(\%)\end{array}$ & $\begin{array}{l}\Delta_{k, n}^{\mathrm{B}} \\
(\%)\end{array}$ & $\begin{array}{c}\text { Peak } \\
k, n\end{array}$ & $\begin{array}{c}f_{k, n}^{\exp } \\
(\mathrm{MHz})\end{array}$ & $\begin{array}{l}f_{k, n}^{\operatorname{sim}, \mathrm{A}} \\
(\mathrm{MHz})\end{array}$ & $\begin{array}{l}f_{k, n}^{\operatorname{sim}, \mathrm{B}} \\
(\mathrm{MHz})\end{array}$ & $\begin{array}{l}\Delta_{k, n}^{\mathrm{A}} \\
(\%)\end{array}$ & $\begin{array}{l}\Delta_{k, n}^{\mathrm{B}} \\
(\%)\end{array}$ \\
\hline 1,1 & 0.943 & 0.886 & 0.927 & -6.0 & -1.6 & 5,4 & 2.712 & 2.607 & 2.722 & -3.9 & 0.4 \\
\hline 1,2 & 1.424 & 1.355 & 1.411 & -4.9 & -0.9 & 5,5 & 2.901 & 2.774 & 2.903 & -4.4 & 0.1 \\
\hline 1,3 & 2.065 & 1.954 & 2.045 & -4.1 & -1.0 & 6,1 & 1.425 & 1.353 & 1.410 & -5.0 & -1.1 \\
\hline 1,4 & 2.717 & 2.613 & 2.722 & -5.4 & 0.2 & 6,2 & 1.537 & 1.465 & 1.531 & -4.7 & -0.4 \\
\hline 1,5 & 2.896 & 2.764 & 2.894 & -4.5 & -0.1 & 6,3 & 1.606 & 1.531 & 1.595 & -4.7 & -0.7 \\
\hline 2,1 & 0.737 & 0.699 & 0.732 & -5.2 & -0.7 & 6,4 & 2.741 & 2.634 & 2.742 & -3.9 & 0.0 \\
\hline 2,2 & 1.418 & 1.351 & 1.408 & -4.7 & -0.7 & 6,5 & 2.910 & 2.784 & 2.913 & -4.3 & 0.1 \\
\hline 2,3 & 1.791 & 1.707 & 1.786 & -4.7 & -0.3 & 7,1 & 0.321 & 0.314 & 0.322 & -2.3 & 0.3 \\
\hline 2,4 & 1.915 & 1.826 & 1.904 & -4.6 & -0.5 & 7,2 & 1.440 & 1.366 & 1.421 & -5.2 & -1.3 \\
\hline 2,5 & 2.942 & 2.811 & 2.942 & -4.5 & -0.0 & 7,3 & 1.561 & 1.480 & 1.542 & -5.2 & -1.2 \\
\hline 3,1 & 0.612 & 0.580 & 0.607 & -5.2 & -0.8 & 7,4 & 2.733 & 2.626 & 2.735 & -3.9 & 0.1 \\
\hline 3,2 & 1.403 & 1.336 & 1.395 & -4.8 & -0.5 & 7,5 & 2.905 & 2.777 & 2.905 & -4.4 & 0.0 \\
\hline 3,3 & 1.489 & 1.420 & 1.480 & -4.6 & -0.6 & 8,1 & 1.438 & 1.361 & 1.416 & -5.4 & -1.6 \\
\hline 3,4 & 1.752 & 1.668 & 1.741 & -4.8 & -0.6 & 8,2 & 1.533 & 1.456 & 1.518 & -5.0 & -1.0 \\
\hline 3,5 & 2.735 & 2.632 & 2.739 & -3.7 & 0.2 & 8,3 & 1.834 & 1.746 & 1.821 & -4.8 & -0.7 \\
\hline $4,1^{*}$ & 0.522 & 0.494 & 0.519 & -5.4 & -0.7 & 8,4 & 2.718 & 2.613 & 2.726 & -3.9 & 0.3 \\
\hline $4,2^{*}$ & 0.544 & 0.525 & 0.547 & -3.4 & 0.7 & 8,5 & 2.903 & 2.782 & 2.910 & -4.2 & 0.2 \\
\hline 4,3 & 1.431 & 1.360 & 1.416 & -5.0 & -1.0 & 9,1 & 1.429 & 1.358 & 1.415 & -4.9 & -1.0 \\
\hline 4,4 & 1.667 & 1.587 & 1.656 & -4.8 & -0.6 & 9,2 & 1.511 & 1.436 & 1.498 & -4.9 & -0.9 \\
\hline 4,5 & 2.725 & 2.621 & 2.729 & -3.8 & 0.2 & 9,3 & 1.765 & 1.679 & 1.751 & -4.9 & -0.8 \\
\hline 5,1 & 1.429 & 1.358 & 1.415 & -4.9 & -1.0 & 9,4 & 2.089 & 1.993 & 2.082 & -4.6 & -0.3 \\
\hline 5,2 & 1.609 & 1.533 & 1.599 & -4.8 & -0.6 & 9,5 & 2.744 & 2.638 & 2.748 & -3.8 & 0.1 \\
\hline 5,3 & 2.104 & 1.998 & 2.088 & -5.0 & -0.7 & & & & & & \\
\hline
\end{tabular}


nominally identical geometry from different parts of the wafer [see Fig. 1(a)], for the approximately 30 peaks that can be easily identified in the range from 0.1 to $10 \mathrm{MHz}$. About $40 \%$ of the devices selected from columns $1-9$ and rows $A-G$ have a short circuit between one or both of the top electrodes and the bottom electrode, so they are discarded from the study. This is the reason that all seven fabricated devices are listed for only column 8 in Table I. We find that the slope of the base level of the phase spectra $\theta(f)$ can vary even for nominally identical devices, as seen in Fig. 3(d). It turns out that this variance is due to spurious charging effects, perhaps due to the floating electrode. This effect can be avoided by briefly short-circuiting the electrodes with a piece of metallic wire, and thus discharging the system. After discharging, the phase spectra become nearly identical for all devices with the same geometry; see Fig. 3(e). When these precautions are taken, the impedance spectra exhibit a large degree of repeatability and reproducibility.

For each of the nine different device geometries among our 35 selected devices, labeled by the column index $k=$ 1-9 in Table I, we measure the resonance frequencies of the five most prominent isolated nonoverlapping resonance peaks, labeled by $n=1-5$, in the impedance-phase spectra $\theta(f)$. This results in $5 \times 35-2=173$ resonance frequencies (for device 4B, we did not measure all five peaks, but only the three highest). In Table II, we list the experimentally determined values of the resonance frequencies, but to avoid showing all 173 of them, we show only the average resonance frequency $f_{k, n}^{\exp }$ of peak $n$ over all devices (row indices $\mathrm{A}-\mathrm{G}$ in Table I) with column index $k$, which results in $9 \times 5=45$ entries.

\section{MODELING AND NUMERICAL SIMULATION}

We want to compare the experimental results for the impedance spectra and resonance frequencies with a numerical simulation. We therefore use the threedimensional model for acoustofluidic systems recently developed by Skov et al. [23]. This model couples the mechanical displacement field $\mathbf{u}$ and the electric potential $\varphi$ in a system driven by a time-harmonic electric potential $\tilde{\varphi}=\varphi_{0} e^{-i \omega t}$, with angular frequency $\omega=2 \pi f$ and frequency $f$, applied to the interfaces between the metal electrodes and the piezoelectric AlN thin-film transducer. This time-harmonic boundary condition excites time-harmonic fields: the electric potential $\tilde{\varphi}(\mathbf{r}, t)$ in the AlN thin-film transducer and the displacement $\tilde{\mathbf{u}}(\mathbf{r}, t)$ in all solids of the system, where

$$
\tilde{\varphi}(\mathbf{r}, t)=\varphi(\mathbf{r}) e^{-i \omega t}, \quad \tilde{\mathbf{u}}(\mathbf{r}, t)=\mathbf{u}(\mathbf{r}) e^{-i \omega t} .
$$

Fields with a tilde are time-harmonic, whereas those without a tilde are complex-valued amplitudes.

The motion of an elastic solid with a given density $\rho$ (in the model this is the glass, the metal electrodes, or the AlN thin-film transducer) is described by the displacement field $\mathbf{u}$, whereas the electrodynamics is described by the electric displacement field $\mathbf{D}$. The governing equations for $\mathbf{u}$ and D are Cauchy's elastodynamic equation and Maxwell's quasi-electrostatic equation, $\boldsymbol{\nabla} \cdot \mathbf{D}=0$ :

$$
-\rho \omega^{2}\left(1+i \Gamma_{\mathrm{sl}}\right) \mathbf{u}=\nabla \cdot \boldsymbol{\sigma}, \quad \nabla \cdot\left[-\left(1+i \Gamma_{\varepsilon}\right) \boldsymbol{\varepsilon} \cdot \nabla \varphi\right]=0 .
$$

TABLE III. Material parameters at $25^{\circ} \mathrm{C}$ for the AIN thin-film transducer and the SLS float-glass substrate. The frequency-dependent damping coefficients $\Gamma_{\mathrm{sl}}(f)$ and $\Gamma_{\varepsilon}(f)$ in the solid and dielectric domain are obtained by fitting to the measured width of the resonance peaks and to the background level, respectively, for devices $\mathrm{C} 2-\mathrm{G} 2$. The longitudinal and transverse sound speed are called $c_{\mathrm{lo}}$ and $c_{\mathrm{tr}}$, respectively. Since we have a thin film, we use $e_{31, f}=e_{31}-\left(C_{13} / C_{33}\right) e_{33}$ instead of $e_{31}$ [24]. $\epsilon_{0}$ is the vacuum permittivity. The tiny

\begin{tabular}{|c|c|c|c|}
\hline Parameter & Value & Parameter & Value \\
\hline \multicolumn{4}{|c|}{ Thin-film aluminum nitride, AIN $[15,25]$} \\
\hline$\rho$ & $3300 \mathrm{~kg} \mathrm{~m}^{-3}$ & $\Gamma_{\mathrm{sl}}$ & 0.0005 \\
\hline$C_{11}$ & $410.2 \mathrm{GPa}$ & $C_{33}$ & $385.0 \mathrm{GPa}$ \\
\hline$C_{12}$ & $142.4 \mathrm{GPa}$ & $C_{44}$ & $122.9 \mathrm{GPa}$ \\
\hline$C_{13}$ & $110.1 \mathrm{GPa}$ & $C_{66}$ & $133.9 \mathrm{GPa}$ \\
\hline$e_{31, f}$ & $-1.05 \mathrm{Cm}^{-2}$ & $e_{15}$ & $-0.39 \mathrm{C} \mathrm{m}^{-2}$ \\
\hline$e_{33}$ & $1.46 \mathrm{C} \mathrm{m}^{-2}$ & $\Gamma_{\varepsilon}(f)$ & $-[(1.43 \mathrm{MHz}+0.98 f) / 1 \mathrm{GHz}]$ \\
\hline$\epsilon_{11}$ & $9.2 \epsilon_{0}$ & $\epsilon_{33}$ & $10.1 \epsilon_{0}$ \\
\hline \multicolumn{4}{|c|}{ SLS float-glass substrate [19] } \\
\hline$\rho$ & $(2522 \pm 15) \mathrm{kg} \mathrm{m}^{-3}$ & & EPFL) \\
\hline E & $(70 \pm 2) \mathrm{GPa}$ & $s$ & $0.23 \pm 0.01$ \\
\hline$C_{11}$ & $81.1 \mathrm{GPa}$ & $C_{44}$ & $28.4 \mathrm{GPa}$ \\
\hline$c_{\mathrm{lo}}=\sqrt{C_{11} / \rho}$ & $5672 \mathrm{~m} \mathrm{~s}^{-1}$ & $C_{12}$ & $24.2 \mathrm{GPa}$ \\
\hline$c_{\mathrm{tr}}=\sqrt{C_{44} / \rho}$ & $3359 \mathrm{~m} \mathrm{~s}^{-1}$ & $\Gamma_{\mathrm{sl}}(f)$ & $f^{-1} 0.018 \mathrm{MHz}$ \\
\hline
\end{tabular}
metal electrodes are not simulated in this study. 
Here, $\Gamma_{\mathrm{sl}}, \Gamma_{\varepsilon} \ll 1$ are weak damping coefficients, $\boldsymbol{\varepsilon}$ is the dielectric tensor, and $\sigma$ is the stress tensor, which is coupled to $\mathbf{u}$ through a stress-strain relation depending on the material-dependent elastic moduli $C_{i k}$.
For the isotropic glass and metal electrodes, the electric field is unimportant, whereas the relation between the stress tensor $\sigma_{i k}$ and the strain components $\frac{1}{2}\left(\partial_{i} u_{k}+\partial_{k} u_{i}\right)$ is given in the compact Voigt representation as

$$
\left(\begin{array}{c}
\sigma_{x x} \\
\sigma_{y y} \\
\sigma_{z z} \\
\sigma_{y z} \\
\sigma_{x z} \\
\sigma_{x y}
\end{array}\right)=\left(\begin{array}{ccc|ccc}
C_{11} & C_{12} & C_{12} & 0 & 0 & 0 \\
C_{12} & C_{11} & C_{12} & 0 & 0 & 0 \\
C_{12} & C_{12} & C_{11} & 0 & 0 & 0 \\
\hline 0 & 0 & 0 & C_{44} & 0 & 0 \\
0 & 0 & 0 & 0 & C_{44} & 0 \\
0 & 0 & 0 & 0 & 0 & C_{44}
\end{array}\right)\left(\begin{array}{c}
\partial_{x} u_{x} \\
\partial_{y} u_{y} \\
\partial_{z} u_{z} \\
\hline \partial_{y} u_{z}+\partial_{z} u_{y} \\
\partial_{x} u_{z}+\partial_{z} u_{x} \\
\partial_{x} u_{y}+\partial_{y} u_{x}
\end{array}\right)
$$

Here, $C_{i k}$ are the elastic moduli, which are listed for SLS float glass in Table III, and $C_{12}=C_{11}-2 C_{44}$.

In the piezoelectric AlN film, the stress tensor $\sigma$ and the electric displacement field $\mathbf{D}$ are given by the strain tensor $\frac{1}{2}\left[\nabla \mathbf{u}+(\nabla \mathbf{u})^{T}\right]$, the electrical field $-\nabla \varphi$, the electromechanical coupling coefficients $e_{i k}$, the elastic moduli $C_{i k}$, and the electric permittivities $\varepsilon_{i k}$ as

$$
\left(\begin{array}{c}
\sigma_{x x} \\
\sigma_{y y} \\
\frac{\sigma_{z z}}{\sigma_{y z}} \\
\sigma_{x z} \\
\sigma_{x y} \\
D_{x} \\
D_{y} \\
D_{z}
\end{array}\right)=\left(\begin{array}{ccc|ccc|ccc}
C_{11} & C_{12} & C_{13} & 0 & 0 & 0 & 0 & 0 & -e_{31} \\
C_{12} & C_{11} & C_{13} & 0 & 0 & 0 & 0 & 0 & -e_{31} \\
C_{13} & C_{13} & C_{33} & 0 & 0 & 0 & 0 & 0 & -e_{33} \\
\hline 0 & 0 & 0 & C_{44} & 0 & 0 & 0 & -e_{15} & 0 \\
0 & 0 & 0 & 0 & C_{44} & 0 & -e_{15} & 0 & 0 \\
0 & 0 & 0 & 0 & 0 & C_{66} & 0 & 0 & 0 \\
\hline 0 & 0 & 0 & 0 & e_{15} & 0 & \varepsilon_{11} & 0 & 0 \\
0 & 0 & 0 & e_{15} & 0 & 0 & 0 & \varepsilon_{11} & 0 \\
e_{31} & e_{31} & e_{33} & 0 & 0 & 0 & 0 & 0 & \varepsilon_{33}
\end{array}\right)\left(\begin{array}{c}
\partial_{x} u_{x} \\
\partial_{y} u_{y} \\
\partial_{z} u_{z} \\
\hline \partial_{y} u_{z}+\partial_{z} u_{y} \\
\partial_{x} u_{z}+\partial_{z} u_{x} \\
\partial_{x} u_{y}+\partial_{y} u_{x} \\
\hline-\partial_{x} \varphi \\
-\partial_{y} \varphi \\
-\partial_{z} \varphi
\end{array}\right) .
$$

Here, the symmetry of AlN leads to $C_{12}=C_{11}-2 C_{66}$.

In terms of volume (mass) ratios, the device consists of $99.8 \%(99.5 \%)$ glass, $0.2 \%(0.3 \%)$ AlN film, and $0.0 \%$ $(0.2 \%)$ metal electrodes. We find that within the numerical uncertainties, the simulation results are unchanged when the metal electrodes and prestress effects in the AlN film are left out. So, to reduce the memory requirements and to speed up the computation times, we leave out the prestress and the tiny metal electrodes in our modeling. Consequently, the boundary conditions for the electric potential $\varphi$ are as follows: (1) on the interface between the bottom electrode and the AIN film, the potential is grounded, $\varphi=0$; (2) on the interface between one half of the split electrode and the AlN film, $\varphi=+\frac{1}{2} V_{0}$; (3) on the interface between the other half of the split electrode and the AlN film, the potential is in antiphase, $\varphi=-\frac{1}{2} V_{0}$; (4) on the side walls of the AlN film, with normal vector $\mathbf{n}$, the no-free-charge condition applies, $\mathbf{n} \cdot \nabla \varphi=0$. For the mechanical displacement field in the solids, a nonstress condition is applied to all external surfaces, $\mathbf{n} \cdot \boldsymbol{\sigma}=\mathbf{0}$.

Once a solution for $\mathbf{u}$ and $\varphi$ is obtained, the electrical impedance $Z$ can be computed in the model as the ratio of the total voltage drop $V_{0}$ to the integral over the electrical current density $\mathbf{J}$ through the surface $\partial \Omega_{1 / 2}$ of one of the halves of the split electrode:

$$
Z=\frac{V_{0}}{I}=\frac{V_{0}}{\int_{\partial \Omega_{1 / 2}} \mathbf{J} \cdot \mathbf{n} d a} .
$$

As there are no free charges in the system, the only contribution to the current density $\mathbf{J}$ is the polarization current density $\mathbf{n} \cdot \mathbf{J}=\mathbf{e}_{z} \cdot \mathbf{J}_{\mathrm{pol}}=\partial_{t} P_{z}=-i \omega\left(D_{z}+\varepsilon_{0} \partial_{z} \varphi\right)$. We compute $D_{z}$ from Eq. (4) and obtain, as in Ref. [26],

$$
Z=\frac{(i / \omega) V_{0}}{\int_{\partial \Omega_{1 / 2}}\left[e_{31}\left(\partial_{x} u_{x}+\partial_{y} u_{y}\right)+e_{33} \partial_{z} u_{z}+\left(\varepsilon_{0}-\varepsilon_{33}\right) \partial_{z} \varphi\right] d a} .
$$

We implement the model of the AlN-thin-film-actuated glass-block devices in the finite-element-method software package COMSOL Multiphysics 5.4 [27] following the procedure described in Ref. [23]. The coupled field equation [Eq. (2)] for the elastic-solid displacement $\mathbf{u}$ and the electric potential $\varphi$ is implemented together with the constitutive equations [Eqs. (3) and (4)] using the weak-form 
interface "PDE Weak Form." Examples of COMSOL scripts with a PDE-weak-form implementation of acoustofluidics are available in Ref. [23] and as Supplemental Material in Ref. [28].

The system geometry described in Sec. II is meshed fairly coarsely because we study the low end of the spectrum, mainly $f<3 \mathrm{MHz}$, where the wavelengths are larger than $1.1 \mathrm{~mm}$. The mesh of the glass block is a free tetrahedral mesh with a maximum element size of $0.2 \mathrm{~mm}$, whereas the AlN thin film is modeled as a swept structured mesh with three mesh layers in the thickness direction and the same element size along the surface as for the glass. We use quadratic Lagrangian test functions and the mesh convergence test described in Ref. [29] to verify that the numerical resolution is satisfactory.

We use an adaptive-step-size algorithm to compute the impedance spectra. Close to a resonance peak, the step size goes down to $15 \mathrm{~Hz}$, but far from resonance peaks, it increases to $1600 \mathrm{~Hz}$. The resonance frequency is determined as the position of the maximum of a Lorentzian fit containing the 11 closest points. A typical short 0.1-3$\mathrm{MHz}$ spectrum contains $450-500$ points, whereas a long $0.1-10-\mathrm{MHz}$ spectrum contains $2500-2800$ points. A short and a long spectrum take around $0 \mathrm{~h} 40 \mathrm{~min}$ and $2 \mathrm{~h} 50 \mathrm{~min}$, respectively, to compute on our HP-G4 workstation with a 3.7-GHz Intel Core i9 7960X-2.8 16-core processor and a memory of 128 GB RAM.

The spatial form of the acoustic mode corresponding to a given simulated resonance peak is revealed by plotting the displacement field $|\mathbf{u}|$. In the Supplemental Material [30], movies of each of the 45 resonance peaks $(k, n)$ listed in Table II are presented.

\section{COMPARING SIMULATION WITH EXPERIMENTAL RESULTS}

We use the numerical model of Sec. V to simulate electrical-impedance spectra $Z^{\text {sim }}(f)$ through Eq. (6). In the following, we compare these simulation results with the experimental spectra $Z^{\exp }(f)$ described in Sec. IV.

Two examples of such a comparison in the frequency range from 0.1 to $3 \mathrm{MHz}$ are seen in Figs. 4(a) and 4(b) for device geometries 2 and 8, respectively, where we plot the resistive part $\operatorname{Re}[Z]$ of the electrical impedance as an alternative way to reveal the resonance peaks, instead of using the phase spectra $\theta(f)$ as in Figs. 3(c) and 3(d). The single red curve in each plot shows the simulation results obtained with the parameter values listed in Table III, whereas the family of gray curves shows the experimental results for all available devices with the relevant geometry. It is seen that the experimental impedance spectra are reproduced fairly well by the simulation regarding the positions of the resonance peaks and their relative heights. We note that, similarly to Fig. 3, the off-resonance levels of the experimental curves (gray) for the real part $\operatorname{Re}[Z]$

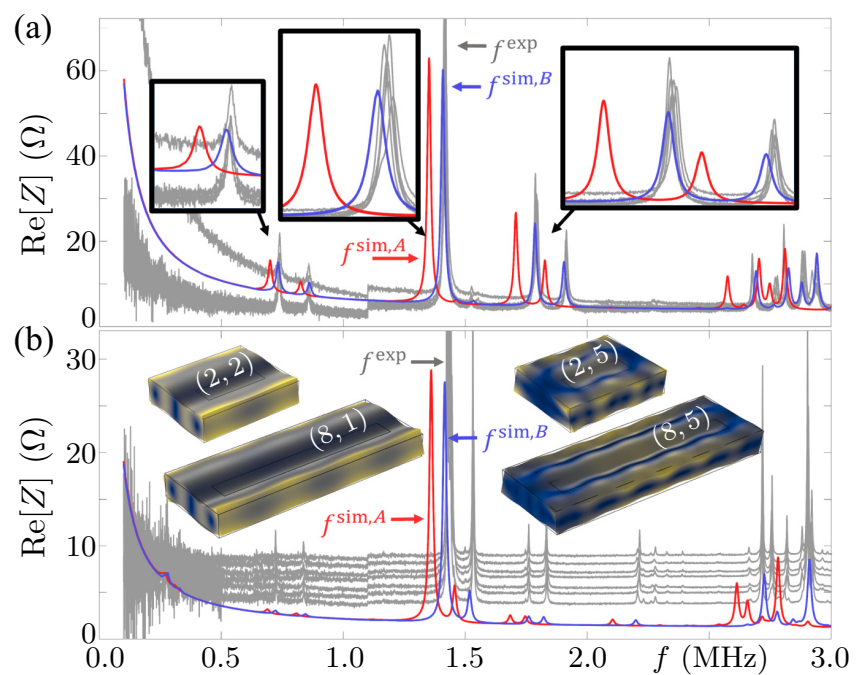

FIG. 4. (a) Resistive real part $\operatorname{Re}[Z]$ of the impedance as a function of frequency $f$ measured for the five devices C2-G2 of Fig. 3(d) (gray curves, $f^{\text {exp }}$ ), and simulated numerically using the parameter values from the literature listed in Table III (red curve, $f^{\text {sim,A }}$ ) and using the fitted parameter values listed in Table IV (blue curve, $f^{\text {sim,B }}$ ). (b) Similar plot of $\operatorname{Re}[Z]$ versus $f$, but for the seven devices A8-G8 of Fig. 3(c). The insets are color plots of the displacement field $|\mathbf{u}|$ for four selected resonance peaks $(k, n)$ from 0 (blue) to maximum (yellow). Movies of the modes of all the simulated resonance peaks in Table II are given in the Supplemental Material [30].

coincide for the devices C2-G2 in Fig. 4(a), which are discharged, while they are displaced for the devices A8-G8 in Fig. 4(b), which are not discharged. Moreover, we note that in Fig. 4(a), we fit the off-resonance level in the numerical model for $Z$ in Eq. (6) using $\Gamma_{\varepsilon}(f)$ given in Table III.

The agreement between the experiments and simulations is quantified in Table II, where it is seen that for the 173 resonance peaks in our study, the relative deviation $\Delta_{k, n}^{\mathrm{A}}$ of each simulated resonance frequency $f_{k, n}^{\text {sim,A }}$ deviates by only a few percent from the experimental value $f_{k, n}^{\exp }$. The mean value and the standard deviation of the mean value of all relative deviations $\Delta_{k, n}^{\mathrm{A}}$ are $(-4.6 \pm 0.1) \%$, which is a fairly good result. However, the simulation results show a statistically significant systematic underestimate of the resonance frequency. This is also clear from the insets in Fig. 4(a) showing enlargements of four resonance peaks: in all cases, the measured (gray) peaks for the seven nominally identical devices fall on top of each other, with variations much smaller than the peak width, whereas, in contrast, the simulated (red) peaks are shifted downward in frequency by a few peak widths.

This systematic shift of the simulated resonance peaks leads us to investigate whether a fitting of the two most significant but not so accurately determined material parameters, the Young's modulus $E$ and Poisson's ratio $s$ of the SLS float-glass substrate, could lead to better numerical 
TABLE IV. Fitted values for Young's modulus $E^{\prime}$, Poisson's ratio $s^{\prime}$, and the damping coefficient $\Gamma_{\mathrm{sl}}$ of SLS float glass for improving the agreement between the experiments and simulations. The values of $C_{11}^{\prime}, C_{12}^{\prime}, C_{44}^{\prime}, c_{\mathrm{tr}}^{\prime}$, and $c_{1 \mathrm{o}}^{\prime}$ are functions of $E^{\prime}$ and $s^{\prime}[33]$.

\begin{tabular}{lccc}
\hline \hline Parameter & Value & Parameter & Value \\
\hline$E^{\prime}$ & $(76 \pm 1) \mathrm{GPa}$ & $s^{\prime}$ & $(0.21 \pm 0.01)$ \\
$C_{11}^{\prime}$ & $85.5 \mathrm{GPa}$ & $C_{44}^{\prime}$ & $31.4 \mathrm{GPa}$ \\
$C_{12}^{\prime}$ & $22.7 \mathrm{GPa}$ & $\Gamma_{\mathrm{sl}}(f)$ & $f^{-1} 0.018 \mathrm{MHz}$ \\
$c_{\mathrm{lo}}^{\prime}=\sqrt{C_{11}^{\prime} / \rho}$ & $5824 \mathrm{~m} \mathrm{~s}^{-1}$ & $c_{\mathrm{tr}}^{\prime}=\sqrt{C_{44}^{\prime} / \rho}$ & $3529 \mathrm{~m} \mathrm{~s}^{-1}$ \\
\hline \hline
\end{tabular}

agreement with the experiments. Indeed, by using a simple stepping procedure, we find that such an improvement is obtained with the substitution

$$
E=70 \mathrm{GPa} \underset{\text { fit }}{\rightarrow}(76 \pm 1) \mathrm{GPa}, \quad s=0.23 \underset{\text { fit }}{\rightarrow} 0.21 \pm 0.01 .
$$

This procedure extends the methods for obtaining the electromechanical coefficients of piezoelectric transducers $[31,32]$ to determining the elastic coefficients of any elastic solid driven by vanishingly small thin-film transducers. The parameter values obtained for the best fitting to the experimental data are summarized in Table IV, and the resonance frequencies obtained from the simulation based on these values are denoted by $f_{k, n}^{\operatorname{sim}, \mathrm{B}}$.

The improved agreement between the simulations and experiments is seen qualitatively in Fig. 4, where the blue curves representing $f_{k, n}^{\text {sim, } \mathrm{B}}$ more accurately reproduce the gray experimental impedance spectra. The improved agreement is also evident from the insets in Fig. 4(a), where the blue curve $f_{k, n}^{\text {sim, B }}$ now falls on top of the gray experimental curves to within a fraction of the peak width. Quantitatively, the improved agreement between the experiments and simulations is seen in Table II, where the relative deviation $\Delta_{k, n}^{\mathrm{B}}$ of each simulated resonance frequency $f_{k, n}^{\text {sim, B }}$ deviates by less than one percent from the experimental value $f_{k, n}^{\exp }$. The mean value and the standard deviation of the mean value of all relative deviations are $\Delta_{k, n}^{\mathrm{B}}=(-0.5 \pm 0.1) \%$, or 9 times better than the first result, $(-4.6 \pm 0.1) \%$, obtained using the manufacturers' parameters.

The improved numerical agreement with the experiments using the fitted values of the Young's modulus and Poisson's ratio for the glass substrate suggests that our numerical-fitting simulation algorithm may be used as a starting point for developing a method for an improved in situ determination of acoustic parameters in the 0.1-10 $\mathrm{MHz}$ range, and possibly at higher frequencies. At the present stage, we do not know whether the relative deviation of roughly $5 \%$ between the literature values in Table III and the fitted values in Table IV is within the uncertainty generated by sample-to-sample variations in the SLS float glass or whether it is a direct result of the fabrication process involving the deposition of the AlN thin-film transducers at $300^{\circ} \mathrm{C}$, which is, however, still in the specified range for stable properties of the float glass studied. The improved agreement between the simulations and experiments also suggests that using impedance measurements coupled with simulations is a promising way to investigate the resonance modes, since there is little ambiguity about which peaks are which. Also, from the measurements shown in Fig. 4, it is clear that by combining simulations with more varied device geometries it will be possible to predict particularly desired resonance frequencies with good accuracy and, consequently, to design appropriate device geometries.

\section{CONCLUSION}

We present the fabrication of $570-\mu \mathrm{m}$-thick millimetersized SLS float-glass blocks with a $1-\mu \mathrm{m}$-thick AlN thinfilm piezoelectric transducer sandwiched between thin metallic electrodes and deposited on the top surface. The electromechanical properties of the devices are characterized by electrical-impedance measurements in the frequency range from 0.1 to $10 \mathrm{MHz}$ with a peak-to-peak voltage of $0.5 \mathrm{~V}$ applied to the electrodes.

We measure the electrical-impedance spectra for 35 different device geometries, all of width $2 \mathrm{~mm}$, but with nine different lengths ranging from 2 to $6 \mathrm{~mm}$ and with two to seven copies of each individual geometry. Each impedance spectrum exhibits many resonance peaks, and we carefully measure the five most prominent ones in each spectrum. Comparing the resulting 173 experimental resonance frequencies with the result of a finite-elementmethod simulation, using material parameter values from the manufacturer, results in an average relative deviation of the 173 simulated resonance frequencies from the experimental values of $(-4.6 \pm 0.1) \%$.

We show that by using the Young's modulus and Poisson's ratio of the SLS float glass as fitting parameters in the simulation, we can reduce the relative deviation of the 173 simulated resonance frequencies from the experimental values by a factor of 9 to $(-0.5 \pm 0.1) \%$. Given a more sophisticated automated fitting procedure, ideally involving more resonance peaks and devices with different electrode configurations for better excitation of other resonance modes than those obtained with the current devices, this result suggests that our numerical fitting procedure could lead to an improved method for in situ determination of the acoustic parameters at ultrasound frequencies of any elastic solid to which a thin-film transducer can be attached.

\section{ACKNOWLEDGMENTS}

This work was supported by the BioWings project funded by the European Union's Horizon 2020 Future 
and Emerging Technologies (FET) program, Grant No. 801267.

[1] R. Ruby, in 2017 IEEE 30th International Conference on MicroElectro Mechanical Systems (MEMS) (IEEE, New York, 2017), p. 308.

[2] M. Dubois and P. Muralt, Properties of aluminum nitride thin films for piezoelectric transducers and microwave filter applications, Appl. Phys. Lett. 74, 3032 (1999).

[3] G. Piazza, P. J. Stephanou, and A. P. Pisano, Piezoelectric aluminum nitride vibrating contour-mode MEMS resonators, J. Microelectromech. Syst. 15, 1406 (2006).

[4] U. Zaghloul and G. Piazza, Highly scalable NEMS relays with stress-tuned switching voltage using piezoelectric buckling actuators, IEEE Trans. Electron. Device 61, 3520 (2014).

[5] N. Sinha, G. E. Wabiszewski, R. Mahameed, V. V. Felmetsger, S. M. Tanner, R. W. Carpick, and G. Piazza, Piezoelectric aluminum nitride nanoelectromechanical actuators, Appl. Phys. Lett. 95, 053106 (2009).

[6] A. De Pastina, D. Maillard, and L. Villanueva, Fabrication of suspended microchannel resonators with integrated piezoelectric transduction, Microelectron. Eng. 192, 83 (2018).

[7] R. H. Olsson III, K. E. Wojciechowski, M. S. Baker, M. R. Tuck, and J. G. Fleming, Post-CMOS-compatible aluminum nitride resonant MEMS accelerometers, J. Microelectromech. Syst. 18, 671 (2009).

[8] H. Zhang, W. Pang, H. Yu, and E. S. Kim, High-tone bulk acoustic resonators on sapphire, crystal quartz, fused silica, and silicon substrates, J. Appl. Phys. 99, 124911 (2006).

[9] J. Masson, G. Martin, R. Boudot, Y. Gruson, S. Ballandras, A. Artieda, P. Muralt, B. Belgacem, and L. Chomeloux, in 2007 IEEE International Frequency Control SymposiumJoint with the 21st European Frequency and Time Forum (IEEE, New York, 2007), p. 741.

[10] T. Fujikura, O. Matsuda, D. M. Profunser, O. B. Wright, J. Masson, and S. Ballandras, Real-time imaging of acoustic waves on a bulk acoustic resonator, Appl. Phys. Lett. 93, 261101 (2008).

[11] P. Muralt, Recent progress in materials issues for piezoelectric MEMS, J. Am. Ceram. Soc. 91, 1385 (2008).

[12] K. M. Howell, W. Bashir, A. De Pastina, R. Matloub, P. Muralt, and L. G. Villanueva, Effect of AIN seed layer on crystallographic characterization of piezoelectric AIN, J. Vac. Sci. Technol., A 37, 021504 (2019).

[13] R. Matloub, A. Artieda, C. Sandu, E. Milyutin, and P. Muralt, Electromechanical properties of $\mathrm{Al}_{0.9} \mathrm{Sc}_{0.1} \mathrm{~N}$ thin films evaluated at $2.5 \mathrm{GHz}$ film bulk acoustic resonators, Appl. Phys. Lett. 99, 092903 (2011).

[14] R. Matloub, M. Hadad, A. Mazzalai, N. Chidambaram, G. Moulard, C. S. Sandu, T. Metzger, and P. Muralt, Piezoelectric $\mathrm{Al}_{1-x} \mathrm{Sc}_{\mathrm{x}} \mathrm{N}$ thin films: A semiconductor compatible solution for mechanical energy harvesting and sensors, Appl. Phys. Lett. 102, 152903 (2013).
[15] Y. Q. Fu, J. K. Luo, N. T. Nguyen, A. J. Walton, A. J. Flewitt, X. T. Zu, Y. Li, G. McHale, A. Matthews, E. Iborra, H. $\mathrm{Du}$, and W. I. Milne, Advances in piezoelectric thin films for acoustic biosensors, acoustofluidics and lab-on-chip applications, Prog. Mater. Sci. 89, 31 (2017).

[16] J.-B. Lee, D.-H. Cho, D.-Y. Kim, C.-K. Park, and J.-S. Park, Relationships between material properties of piezo-electric thin films and device characteristics of film bulk acoustic resonators, Thin Solid Films 516, 475 (2007).

[17] M. Dubois and P. Muralt, Stress and piezoelectric properties of aluminum nitride thin films deposited onto metal electrodes by pulsed direct current reactive sputtering, J. Appl. Phys 89, 6389 (2001).

[18] C. K. Lee, F. Placido, S. Cochran, and K. J. Kirk, in Proceedings, 2002 IEEE Ultrasonics Symposium, 2002, Vol. 2 (IEEE, New York, 2002), p. 1119.

[19] Float glass substrates, [soda-lime-silicate glass: $\mathrm{SiO}_{2}$ (70.8\%), $\mathrm{Na}_{2} \mathrm{O}(13.9 \%), \mathrm{CaO}(8.4 \%), \mathrm{MgO}(4.4 \%), \mathrm{Al}_{2} \mathrm{O}_{3}$ (1.5\%), $\left.\mathrm{K}_{2} \mathrm{O}(0.4 \%), \mathrm{SO}_{3}(0.3 \%), \mathrm{Fe}_{2} \mathrm{O}_{3} \quad(0.08 \%)\right]$, SCHOTT, SCHOTTSuisse SA, 2 Rue Galilée, Yverdon, Switzerland, https:/www.epfl.ch/research/facilities/cmi/ wp-content/uploads/2020/05/float_glass.pdf (accessed 16 November 2020).

[20] A. Migliori, J. Sarrao, W. Visscher, T. Bell, M. Lei, Z. Fisk, and R. Leisure, Resonant ultrasound spectroscopic techniques for measurement of the elastic-moduli of solids, Physica B 183, 1 (1993).

[21] M. Radovic, E. Lara-Curzio, and L. Riester, Comparison of different experimental techniques for determination of elastic properties of solids, Mater. Sci. Eng. A-Struct. 368, 56 (2004).

[22] A. Cachiaras, L. Gilde, J. Swab, P. Patel, and G. Quinn, Soda-lime-silicate float glass: A property comparison, US Army Research Laboratory, ARL, Aberdeen Proving Ground, MD 21005-5069, USA (2017), https://www.resear chgate.net/publication/320871872 (accessed 16 November 2020).

[23] N. R. Skov, J. S. Bach, B. G. Winckelmann, and H. Bruus, 3D modeling of acoustofluidics in a liquid-filled cavity including streaming, viscous boundary layers, surrounding solids, and a piezoelectric transducer, AIMS Math. 4, 99 (2019).

[24] P. Muralt, J. Conde, A. Artieda, F. Martin, and M. Cantoni, Piezoelectric materials parameters for piezoelectric thin films in GHz applications, Int. J. Microw. Wirel. Technol. 1, 19 (2009).

[25] M. A. Caro, S. Zhang, T. Riekkinen, M. Ylilammi, M. A. Moram, O. Lopez-Acevedo, J. Molarius, and T. Laurila, Piezoelectric coefficients and spontaneous polarization of ScAlN, J. Phys.-Condens. Matter 27, 245901 (2015).

[26] N. R. Skov, P. Sehgal, B. J. Kirby, and H. Bruus, ThreeDimensional Numerical Modeling of Surface-AcousticWave Devices: Acoustophoresis of Micro- and Nanoparticles Including Streaming, Phys. Rev. Appl. 12, 044028 (2019).

[27] COMSOL Multiphysics 5.4 (2018), http://www.comsol. com.

[28] P. B. Muller and H. Bruus, Theoretical study of time-dependent, ultrasound-induced acoustic streaming in microchannels, Phys. Rev. E 92, 063018 (2015). 
[29] P. B. Muller, R. Barnkob, M. J. H. Jensen, and H. Bruus, A numerical study of microparticle acoustophoresis driven by acoustic radiation forces and streaminginduced drag forces, Lab Chip 12, 4617 (2012).

[30] See Supplemental Material at http://link.aps.org/supple mental/10.1103/PhysRevApplied.16.014014 for movies of the time-dependent displacement field $|\boldsymbol{u}|$ for each of the 45 resonance peaks $(k, n)$ listed in column $f_{k, n}^{\text {sim, B }}$ of Table II.

[31] T. Lahmer, M. Kaltenbacher, B. Kaltenbacher, R. Lerch, and E. Leder, FEM-based determination of real and complex elastic, dielectric, and piezoelectric moduli in piezoceramic materials, IEEE Trans. Ultrason. Ferroelectr. Freq. Control 55, 465 (2008).

[32] C. Y. Kiyono, N. Perez, and E. C. N. Silva, Determination of full piezoelectric complex parameters using gradientbased optimization algorithm, Smart Mater. Struct. 25, 025019 (2016).

[33] J. T. Karlsen and H. Bruus, Forces acting on a small particle in an acoustical field in a thermoviscous fluid, Phys. Rev. E 92, 043010 (2015). 